\title{
¿EXISTE UNA TEORÍA EDUCATIVA TRAS LA CONVENCIÓN DE LOS DERECHOS DEL NIÑO?
}

Resumen. La Convención de los Derechos del Niño es sin duda el instrumento jurídico más importante y complejo que existe sobre la infancia en el ámbito del Derecho Internacional. Esta complejidad se acentuada por cuestiones de índole pedagógica y psicológica tales como el interés superior del niño o el concepto de evolución de las facultades del niño. En efecto, dichos conceptos no pueden ser interpretados, ni desarrollados únicamente desde una perspectiva jurídica. Así, el ámbito de la Teoría de la Educación se nos revela como un espacio pedagógico privilegiado no sólo para realizar una lectura educativa y crítica de la Convención, sino sobre todo para explorar el alcance de dichas nociones en relación a los principios de autonomía y cuidado implícitos a lo largo de la Convención y las posibles vías para su conciliación.

Palabras clave: Derechos del niño; interés superior del niño; evolución de las facultades del niño; enfoque relacional; autonomía; cuidado.

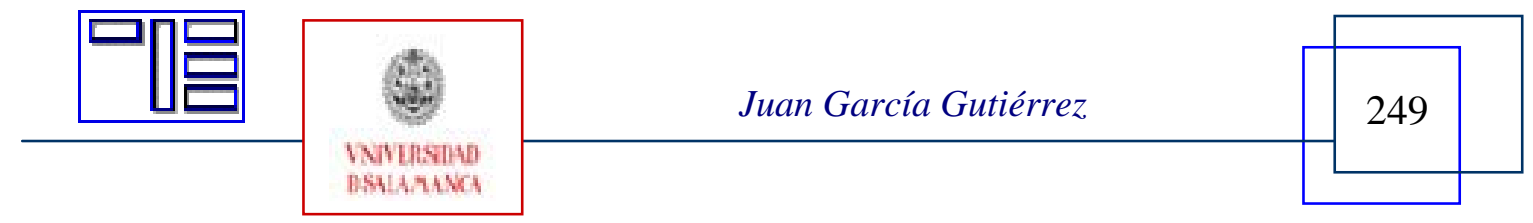




\section{IS THERE AN EDUCATIONAL THEORY AFTER THE CONVENTION ON THE RIGHTS OF THE CHILD?}

Abstract. The Convention on the Rights of the Child is undoubtfully the most important and complex law instrument about childhood in the frame of International Law. This Complexity is highlighted by issues of pedagogical and psychological nature, such as the best interests of the child or the concept of the child's powers development. Indeed, such concepts cannot be interpreted, nor developed, solely in the light of law. Thus, the grounds of the Educational Theory are revealed as a privileged pedagogical ground, not only to achieve an educative and critic comprehension of the Convention, but principally to explore the reach of those notions in relationship with the principles of autonomy and care, both implicit in the Convention, and the possible means for its conciliation.

Keywords: Rights of the Child, The best interests of the child, Child's powers development, Relational scope, Autonomy, Care.

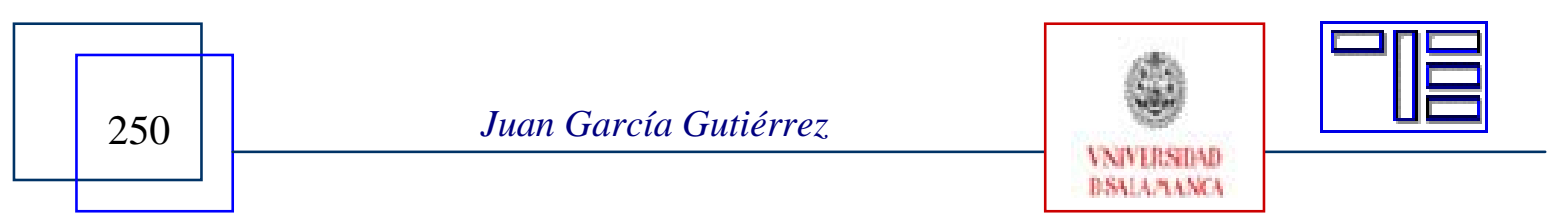




\section{Y AT-IL UNE THÉORIE ÉDUCATIVE APRÈS LA CONVENTION SUR LES DROITS DE L'ENFANT?}

Sommaire. La Convention relative aux droits de l'enfant undoubtfully est la plus importante et complexe instrument de droit sur l'enfance dans le cadre du droit international. Cette complexité est mise en évidence par des questions de nature pédagogique et psychologique, tels que l'intérêt supérieur de l'enfant ou de la notion de l'enfant pouvoirs du développement. En fait, ces concepts ne peuvent pas être interprétées, ni mis au point, uniquement à la lumière du droit. Ainsi, les motifs de la Théorie de l'éducation sont révélés comme un terrain pédagogique privilégié, non seulement pour parvenir à une compréhension critique éducative et de la Convention, mais prin-pally d'explorer la portée de ces notions en relation avec les principes de l'au-tonomie et Soins, à la fois implicite dans la Convention, et les moyens possibles pour sa conciliation.

Mots-clés: droits de l'enfant, l'intérêt supérieur de l'enfant, Child pouvoirs du développement, la portée relationnel, autonomie, les soins.

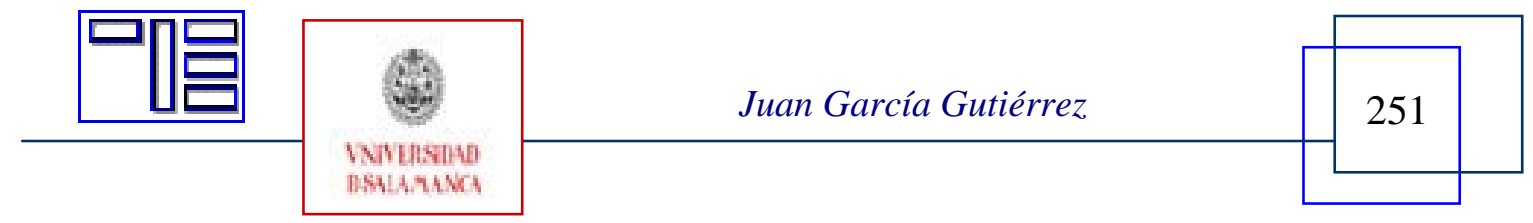




\title{
¿EXISTE UNA TEORÍA EDUCATIVA TRAS LA CONVENCIÓN DE LOS DERECHOS DEL NIÑO?
}

\author{
Juan García Gutiérrez \\ juangarcia.edu@gmail.com \\ Instituto de Estudios Educativos y Sociales, Madrid
}

\begin{abstract}
"Reconociendo que el niño, para el pleno y armonioso desarrollo de su personalidad, debe crecer en el seno de la familia, en un ambiente de felicidad, amor y comprensión”. Preámbulo, Convención de los derechos del niño de 1989.
\end{abstract}

\begin{abstract}
“Nuestra esperanza siempre está en lo nuevo que trae cada generación; pero precisamente porque podemos basar nuestra esperanza tan sólo en esto, lo destruiríamos todo si tratáramos de controlar de ese modo a los nuevos, a quienes nosotros, los viejos, les hemos dichos cómo deben ser. Precisamente por el bien de lo que hay de nuevo y revolucionario en cada niño, la educación ha de ser conserva-

dora".
\end{abstract}

H. Arendt, Entre el pasado y el futuro.

\section{1.- INTRODUCCIÓN}

La Convención de los Derechos del Niño (CDN) es sin duda uno de los instrumentos jurídicos más complejos que existen en el Derecho Internacional. Esta complejidad es acentuada por cuestiones de índole pedagógica y psicológica tales como el interés superior del niño (ISN) o el concepto de evolución de las facultades del niño. En efecto, dichos conceptos no pueden ser interpretados, ni desarrollados únicamente desde una perspectiva jurídica. Así el ámbito de la Teoría de la Educación se nos revela como un espacio pedagógico adecuado para hacer una lectura educativa y crítica de la Convención de los Derechos del Niño sobre todo atendiendo a estas dos nociones ${ }^{1}$.

En una lectura pedagógica de la Convención lo primero que llama la atención al es la tensión que existe a lo largo de su articulado entre los principios de autonomía y cuidado. La tensión es expresión no sólo de diversas concepciones o teorías educativas, sino también de factores jurídicos y políticos, como la fractura internacional del momento. En efecto, aún se encuentran en la Convención rastros de la característica división de bloques que afectó durante muchos años a los textos internacionales de derechos

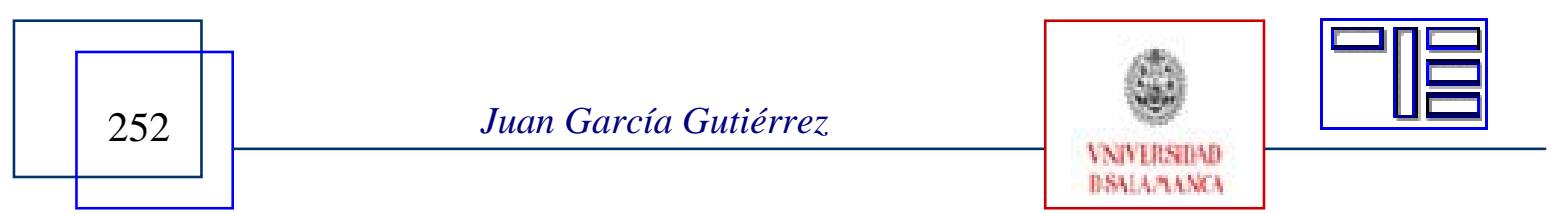


humanos debido a la “Guerra fría”. Quizá el ejemplo más significativo de esta tensión lo constituye la noción de interés superior del niño (ISN). En efecto, en muchas ocasiones el ISN puede significar autonomía pero en otras su interpretación exige el cuidado de la infancia. Por ello y ante coyunturas socio-políticas en las que aparecen intereses y posturas contrapuestas se hace necesario una lectura pedagógica que ayude a identificar cuando el interés superior del niño es invocado no desde su propio interés sino desde otro tipo de intereses no tan superiores.

El desarrollo del ISN aparece vinculado, en el ámbito interno, al derecho de familia, y en concreto, a la función de mediación entre los intereses del padre y de la madre sobre la custodia de sus hijos en casos de separación y divorcio, o en los casos de adopción, etc. También ha sido importante la evolución que ha experimentado el derecho penal para los menores. En el plano internacional, se reconoce por primera vez su carácter jurídico en la Convención de los Derechos del Niño de 1989, situándolo como principio que debe regir la interpretación y aplicación de los demás derechos recogidos. El Interés Superior del Niño es actualmente la máxima expresión jurídica de la protección que los adultos ofrecen a las nuevas generaciones. Protección, que en ocasiones que se concreta en cuidados y protección especial, tanto antes como después del nacimiento (tal y como indicaba la Declaración del '59); o bien en el desarrollo y el respeto de la propia autonomía personal.

Sin embargo, que el ISN haya llegado a convertirse en la clave de bóveda de la CDN no se puede explicar únicamente desde una perspectiva jurídica. En efecto, esta evolución jurídica se explica principalmente por el desarrollo y la evolución acaecidos sobre todo en el ámbito educativo y de la psicología infantil. En efecto, la consideración del niño como un ser autónomo y sujeto de derechos, tal cual lo propone la Convención, es el resultado de una progresiva transformación educativa, de un giro copernicano (M. Soëtard, 1994; E. Becchi, 1998) que comienza a fraguarse en el siglo XVIII, con la publicación en 1762 del Emilio, de Rousseau y tendrá su apogeo con los movimientos de renovación pedagógica de finales del S. XIX. De hecho es necesario recordar la figura del educador polaco Janusz Korczak ${ }^{2}$ ya que en 1978, conmemorando el aniversario de su nacimiento, se celebró el Año Internacional del Niño, y a propuesta de la delegación

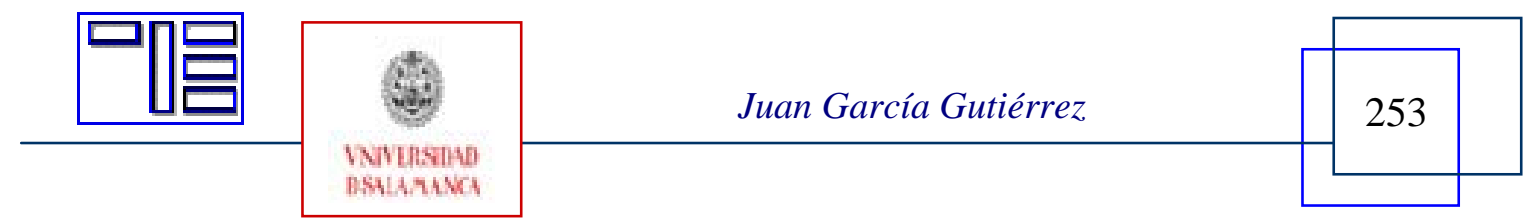


polaca comenzaron los trabajos preparatorios que finalizarían con la firma de la Convención de Derechos del Niño en 1989.

\section{2.- "LA HUMANIDAD DEBE DAR A LA INFANCIA LO MEJOR DE SÍ MIS- MA”}

Después de la Primera Guerra Mundial, la recientemente creada Sociedad de Naciones, consciente del sufrimiento que la “Gran Guerra” había inflingido a los niños y de la importancia de éstos para el futuro de la humanidad, se estableció una Declaración (1924), conocida como “Declaración de Ginebra” donde se expresaba brevemente las principales preocupaciones de la sociedad internacional sobre la infancia. Esta Declaración contenía cinco principios: el niño debe ser puesto en condiciones de desarrollarse normalmente desde el punto de vista material y espiritual; el niño hambriento debe ser alimentado, el niño enfermo debe ser atendido, el niño deficiente debe ser ayudado, el niño desadaptado debe ser radicado, el huérfano y el abandonado deben ser recogidos y ayudados; el niño debe ser el primero en recibir socorro en caso de calamidad; el niño deber ser puesto en condiciones de ganarse la vida y debe ser protegido de cualquier explotación; el niño debe ser educado inculcándose el sentido del deber que tiene de poner sus mejores cualidades al servicio del prójimo. La forma en que esta Declaración tiene de enunciar el ISN es a través de afirmar que la "la humanidad debe dar al niño lo mejor de si misma”.

Otro paso importante hacia la institucionalización del ISN será dado en 1959 cuando la Asamblea General de las UN, después del desastre de la IIGM, proclame una nueva Declaración de Derechos del Niño (Resolución 1386, XIV de 20.11.59). De esta forma, siguiendo la línea iniciada ya en 1924 se establecen los derechos del niño a través de diez principios. En el segundo principio se afirmará por vez primera de forma explícita el ISN en un texto internacional de derechos humanos. En efecto, tras la IIGM aparece un momento político en el que los grandes líderes preocupados por la paz vuelven su mirada a la infancia y a la educación. En primer lugar, hay que destacar la Carta de las Naciones Unidas (1945), nosotros los pueblos de las Naciones Unidad, decididos a salvar a las futuras generaciones del flagelo de la guerra. El sentido y el alcance de

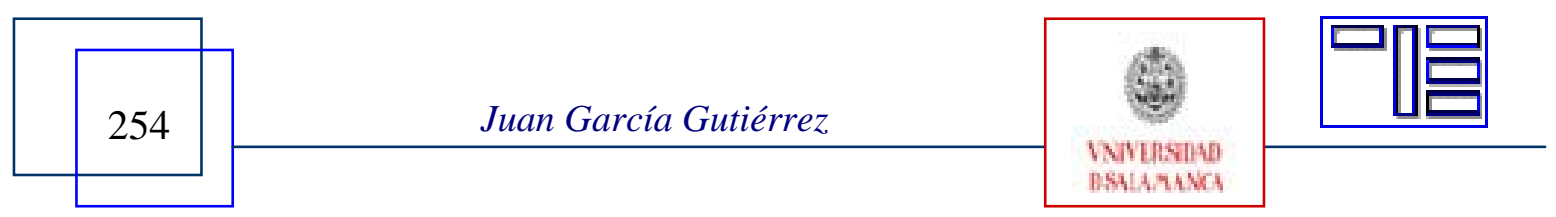


esta afirmación hay que leerlo estrechamente ligado a esa otra proclamación hecha por la Sociedad de Naciones, la humanidad debe a la infancia lo mejor de sí misma. Estas dos proclamaciones nos hacen vislumbrar la importancia de la infancia en el futuro de la humanidad.

Aun en el contexto de la Guerra Fría, otros textos relevantes para comprender el desarrollo del ISN son aquellos que se refieren, por una parte a los derechos civiles y políticos, y por otra, a los derechos económicos, sociales y culturales. En efecto, en el Pacto Internacional de Derechos Civiles y Políticos (1966) en su artículo 27 establece, entre otras cosas, que todo niño tiene derecho, (...) a las medidas de protección que su condición de menor requiere, tanto por parte de su familia como de la sociedad y del Estado. Por otra parte, el Pacto Internacional de Derechos Económicos, Sociales y Culturales (1966) en el marco de la protección de la familia establece en relación a los niños que los Estados deben adoptar medidas especiales de protección y asistencia en favor de todos los niños y adolescentes, sin discriminación alguna por razón de filiación o cualquier otra condición. Debe protegerse a los niños y adolescentes contra la explotación económica y social. Su empleo en trabajos nocivos para su moral y salud, o en los cuales peligre su vida o se corra el riesgo de perjudicar su desarrollo normal, será sancionado por la ley (...) (art. 10.3).

El año 1979 será decisivo para la institucionalización del ISN por dos motivos principales. En primer lugar, a través de la Convención sobre la eliminación de todas las formas de discriminación contra la mujer (1979) aparece por primera vez afirmado con valor jurídico el principio de ISN, caracterizado como la consideración primordial (art. 5b; 16b), y en segundo lugar, se inician los travaux préparatoires que darán origen diez años más tarde al texto definitivo de la Convención. El proceso de institucionalización está en marcha.

Por otra parte, y descendiendo a los ámbitos regionales, en Europa cabe destacar el Convenio Europeo para la Protección de los Derechos Humanos y las Libertades Fundamentales $(1950)^{3}$. En otros contextos religioso-culturales, como por ejemplo África, el Islam, o América se han desarrollado también instrumentos propios para la protección y promoción de los derechos humanos, donde también aparecen consideraciones

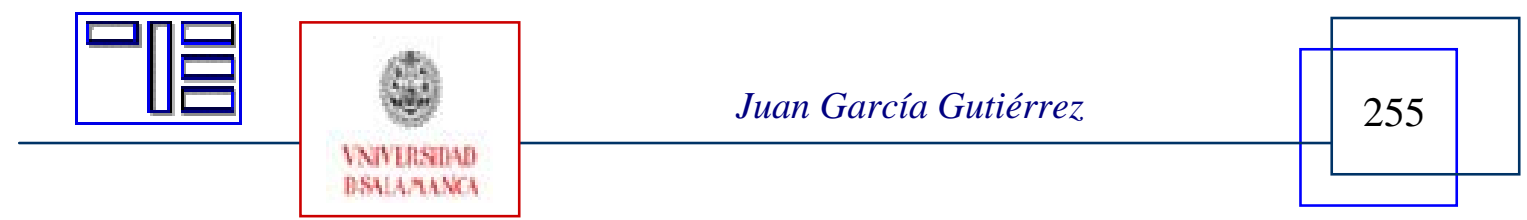


sobre el cuidado y las atenciones a la infancia, siempre en el marco de la protección de la familia, o de los derechos de la mujer durante el embarazo ${ }^{4}$. Una particular atención merece la Carta Africana de Derechos del Hombre y de los Pueblos (1981) donde junto a los derechos se fijan los deberes de las personas, y así se afirma, por ejemplo, el deber del niño de respetar en cada momento a sus padres, nutrirlos y asistirlos en caso de necesidad (art. 29.1) $)^{5}$. Este artículo, aparentemente anecdótico, es significativo por cuanto viene a subrayar el carácter relacional que tienen los derechos humanos, y en concreto también los deberes del niño respecto de su familia. Este carácter relacional de los derechos del niño es algo que en ocasiones se echa de menos en la CDN, donde predomina una visión individualista, casi solipsista, de los derechos del niño.

\section{3.- LA CONVENCIÓN DE LOS DERECHOS DEL NIÑO DE 1989}

Institucionalizar (del latín institutio -onis), significa tanto “dar estatuto de legalidad”, como hacer de algo una “institución”. Una institución, designa tanto la acción como la cosa fundada. Por tanto, sólo podríamos hablar de institucionalización del ISN cuando se producen estas dos condiciones. De un lado, cuando se obtiene el estatuto de legalidad, que sólo llega cuando los Estados miembros de la comunidad internacional se obligan en su cumplimiento, de manera que dotan al ISN de valor jurídico a través de la Convención ${ }^{6}$. Por otro, podemos hablar también de institucionalización en el sentido que se han desarrollado instituciones que velarán por el cumplimiento de este principio. En efecto, tanto el Comité de los Derechos del Niño como UNICEF son dos instituciones que tienen en el ISN su razón de ser.

En palabras de los profesor Ph. Alston \& Steiner (2000: 511), la Convención de Derechos del Niño representa "the most comprehensive single treaty ever to appear in the field of human rights”. La Convención posee la estructura clásica de los textos de derechos humanos de las NU. Esta formada por un Preámbulo y 54 artículos. En el Preámbulo se vienen a recordar los antecedentes más importantes y también los motivos que la hacen necesaria. Entre los artículos se pueden destacar, sobre todo aquellos que constituyen los principios rectores de la Convención, esto es: la no-discriminación (art. 2); el interés superior del niño (art. 3); el derecho intrínseco a la vida y a la supervivencia (art.

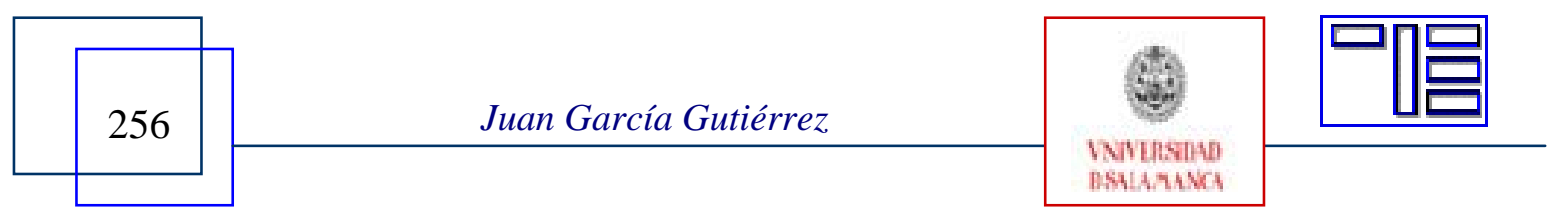


6) y la participación (art. 12). Del artículo 42 a 45 se establecen la obligaciones para los Estados parte, y en los artículos finales, el 46 al 54, son de índole más técnica, sobre la entrada en vigor, el proceso de ratificación del Tratado, etc.

En la CDN el ISN viene “institucionalizado", , de tres formas o maneras principales, bien como una consideración preeminente, bien como la consideración preeminente, o bien como preocupación fundamental (en la exégesis del ISN veremos el porqué de la diversidad de redacciones y sus implicaciones para la comprensión correcta del principio).

“En todas las medidas concernientes a los niños que tomen las instituciones públicas o privadas de bienestar social, los tribunales, las autoridades administrativas o los órganos legislativos, una consideración primordial a que se atenderá será el interés superior del niño (art. 3.1)”.

“Los Estados Partes pondrán el máximo empeño en garantizar el reconocimiento del principio de que ambos padres tienen obligaciones comunes en lo que respecta a la crianza y el desarrollo del niño. Incumbirá a los padres o, en su caso, a los representantes legales la responsabilidad primordial de la crianza y el desarrollo del niño. Su preocupación fundamental será el interés superior del niño (art. 18.1)”.

“Los Estados Partes que reconocen o permiten el sistema de adopción cuidarán de que el interés superior del niño sea la consideración primordial (art. 21”.

\section{4.- EL PROBLEMA DE LA CONCEPTUALIZACIÓN DEL ISN: INDETERMI- NACIÓN Y RELATIVISMO CULTURAL}

Como suele decirse, “necesitamos de las definiciones como necesitamos los relojes, y el más imperfecto de ellos e siempre mejor que su ausencia”. Definir significa señalar los límites de algo, esto es, lo que está dentro y lo que queda fuera. Obviamente algunos conceptos son más difíciles de definir que otros, en más, si lo que se trata de definir no es una palabra sino tres presentes en el mismo concepto, y además se debe traducir a otros idiomas el problema es casi irresoluble. En efecto, esto es lo que sucede con el término que tenemos entre manos, "the best interest of the child".

Una problemática a la que se enfrenta el principio de ISN en la actualidad es la cuestión de la indeterminación. Dicho problema ha surgido principalmente en el campo jurí-

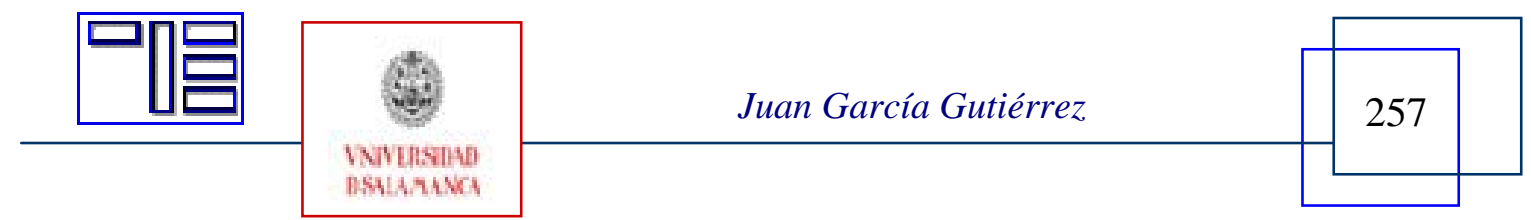


dico, y dejando a un lado consideraciones más técnicas ${ }^{8}$ en la práctica significa que en los asuntos que atañen a la infancia y en los que no existe unanimidad o consenso sobre lo qué es, o sobre lo que constituye su interés superior, el ISN es utilizado para fundamentar cualquier posición, incluso posiciones opuestas. No sólo la diferencia cultural genera interpretaciones controvertidas a cerca de lo que es "bueno" (mejor) o "malo" (peor) para el niño, sino que la confluencia dentro de una misma sociedad de ideologías distintas convierte en muchos casos el ISN en un campo de batalla (I. N. Voss, 1993), o en un principio ideológico, que en última instancia vendrá a legitimar y respaldar las medidas que se adopten sobre la infancia desde el poder, sin entrar realmente a cuestionarse si éstas satisfacen el ISN o satisfacen otro tipo de intereses quizá legítimos, pero no tan superiores.

Pero veamos en qué sentido podemos entender la cuestión de la indeterminación. A nuestro juicio, podemos individuar dos tipos de indeterminación en el discurso sobre el ISN. Por un lado, no encontramos con una indeterminación que podríamos denominar como extrínseca, esto es, la variedad de circunstancias a las que se aplica el principio, y la multiplicidad de contextos y situaciones hacen difícil fijar un contenido concreto ya que éste, en última instancia, siempre es algo individualizado para situación concreta. La indeterminación no vendría por la naturaleza del principio como por la variabilidad de circunstancias a las que debe ser aplicado. Este tipo de indeterminación, pese a ser problemática, no lo es tanto como aquella indeterminación que podríamos denominar como intrínseca. En efecto, por otro lado, podemos individuar también un tipo de indeterminación interna, que partiendo de la flexibilidad necesaria para la aplicación del principio a situaciones concretas afirma la imposibilidad de que éste pueda tener un contenido concreto y universal.

En suma, mientras que en unos casos, la indeterminación estaría fuera del principio, esto es, en su aplicación, en otros, la indeterminación constituiría la naturaleza propia de la noción de ISN, y por lo tanto no sería un principio válido para ser desarrollado en el contexto de la CDC, ya que al ser un principio fácilmente instrumentalizable por la ausencia de contenido concreto, paradójicamente podría ser utilizado en detrimento de los propios derechos que la Convención trata de reconocer. Mientras que en algunos casos

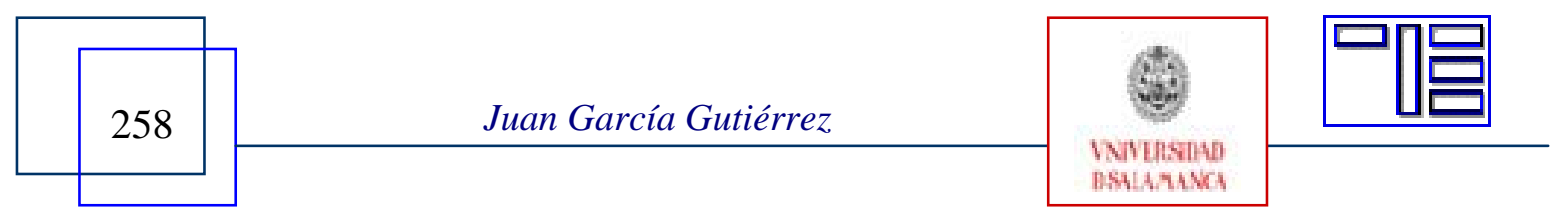


existe una dificultad objetiva en la aplicación de estándares sobre qué constituye lo mejor para el niño, para la infancia, dada la complejidad creciente de los contextos en los que el principio viene a ser aplicado, en otros, no son las circunstancias lo que lo vuelven indeterminado, sino la imposibilidad de encontrar un contenido normativo preciso; en última instancia, no hay nada que nos diga qué es lo mejor para el niño o para la infancia independientemente de la cultura, el contexto social, el ámbito geográfico, etc ${ }^{9}$.

Sobre la relación entre el relativismo cultural y el ISN resulta especialmente interesante el proyecto que dirigió el profesor Ph. Alston (1994), a través del "Unicef Internacional Child Development Centre” de Florencia, que llevaba por título "Best Interest of the Child Project”. El estudio analiza las distintas implicaciones que tienen la aplicación y desarrollo del ISN en contextos geográficos y culturales diversos (tales como Zimbawe, Francia, Japón, el mundo árabe, etc.). Más allá de la complejidad del tema los autores llegan a una conclusión aparentemente sencilla: a pesar de la importancia y diversidad manifiesta de los valores culturales, de las costumbres, de las tradiciones jurídicas, etc. éstas deben ceder cuando entran en conflicto con un derecho humano, y en este caso, cuando van en detrimento del ISN. Estos problemas ya estaba presentes en muchas de las discusiones sobre la conceptualización del ISN, tal y como lo pone de manifiesto la observación de uno de los relatores del grupo de trabajo sobre que la frase (o sea, “el interés superior del niño”) es inherentemente subjetiva y su interpretación sería inevitablemente dejada al juicio de la persona, la institución o la organización que lo aplique (E/CN.4/1989/48, parr. 120)”.

En fin, frente a estas indeterminaciones (intrínseca y extrínseca) como ya hemos visto, además podemos incluir otro problema, podríamos decir, de tipo lingüístico, que dificulta la comprensión del principio. En efecto, como ya hemos visto el ISN aparece formulado de distintas formas en la Convención. Este hecho no es accidental y nos lleva a cuestionarnos el significado y las implicaciones que dichas redacciones tienen para la correcta interpretación del principio, qué sentido pueden tener tanto la omisión, como la introducción de determinadas palabras en la redacción del artículo.

\section{1.- ¿Es posible un sentido unitario en el ISN (1979-1989)?}

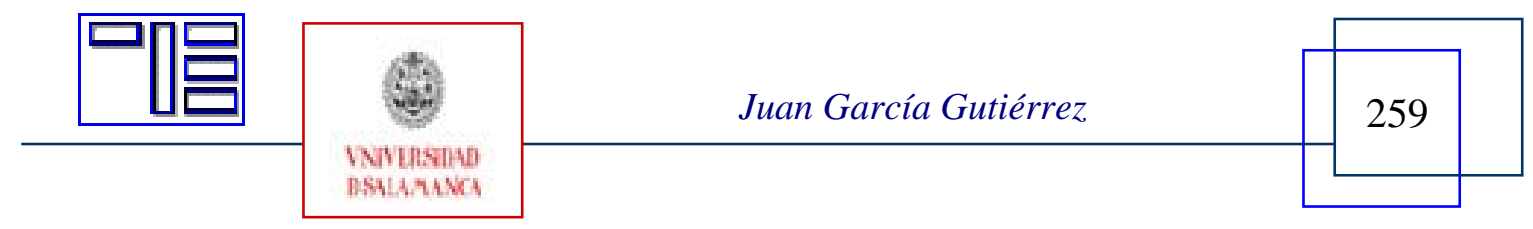


Realizar una exégesis del principio de ISN no resulta fácil, sobre todo porque ya en el inicio los trabajos preparatorios no fueron fáciles (S. Detrick, 1992; L. Chen, 1989; J. S. Cerda, 1990; D. McGoldrick, 1991). El primer borrador aparece a propuesta de Polonia y básicamente consistía en una adaptación de los principios ya contenidos en la Declaración de 1959. A lo largo de estos trabajos se han sucedido diversas redacciones del principio de ISN. La primera aparece en 1978, en el primer borrador a propuesta polaca. En esta redacción se incluye sin modificaciones la formulación inicial del ISN como principio segundo de la Declaración de 1959,

“El niño gozará de una protección especial y dispondrá de oportunidades y servicios, dispensado todo ello por la ley y por otros medios, para que pueda desarrollarse física, mental, moral, espiritual y socialmente en forma saludable y normal, así como en condiciones de libertad y dignidad. Al promulgar leyes con este fin, la consideración fundamental a que se atenderá será el interés superior del niño (Commission on Human Rights res. 20 (XXXIV) de 8.3.1978)”.

Sin embargo, el documento de trabajo de 1980 ya recoge una redacción diversa a la original,

"En todas las medidas concernientes a los niños, asumidas por sus padres, tutores legales, instituciones sociales o públicas, y en particular las autoridades administrativas y los tribunales de justicia, el interés superior del niño será la consideración fundamental (E/CN.4/1349)”.

A propuesta de la delegación americana en las siguientes redacciones se sustituye el término “consideración fundamental”, paramount consideration, por "una consideración primordial”, a primary consideration (E/CN.4/L.1575, pp. 3-7). En efecto, en los trabajos preparatorios podemos apreciar básicamente dos tendencias, una que entiende el “interés superior” como "paramount considerarion” [consideración fundamental], pronto desestimada por considerarse en exceso amplia, y una segunda, tendente a considerarlo como "primary consideration" [consideración primordial], que en última instancia, es la que ha logrado la mayor adhesión. Otra propuesta que merece ser mencionada, aunque no tuvo éxito, fue la planteada por la delegación belga, que proponía

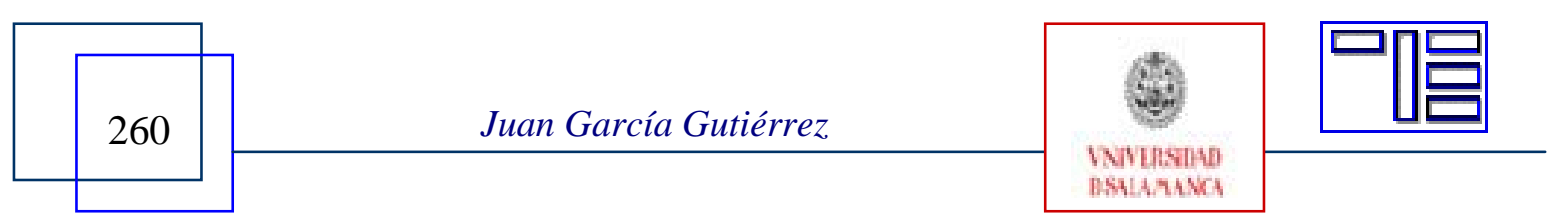


una redacción alternativa del ISN en la que éste no se mencionaba de forma explícita (E/CN.4/1983/62, anex II).

En efecto, al optar por el término “a primary consideration” se está facilitando interpretación más flexible de los intereses en juego. Por ejemplo, como puso de manifiesto uno de los Relatores del Grupo de Trabajo, el interés del niño no debería ser la consideración primordial en todos los casos, ya que otras partes podrían tener igual o superior interés legal en algunas situaciones, como puede ser el caso de una emergencia medica durante el nacimiento (E/CN.4/L.1575, parr. 24). La redacción final del ISN como "a primary consideration" no hace sino aclarar el tipo de interpretación y el alcance que debe darse al principio. La preferencia manifestada por los redactores de la Convención al optar por este tipo de redacción del principio, más formal que sustantiva, indica que el ISN no puede prevalecer ante otros intereses como "la consideración primordial”, sino que debe ser considerado como un factor más, relevante eso sí, pero junto a otros intereses en juego.

Durante la revisión técnica en 1989 del borrador de la Convención se introdujo una nueva modificación en la consideración del ISN, que pasaba a ser “the primary consideration (E/CN.4/1989/48, parr.117)”. La razón para esta nueva modificación era dotar al principio de una mayor congruencia, ya que había sido introducido en otros Tratados internacionales con esa misma redacción (como por ejemplo, la Convención sobre la eliminación de todas las formas de discriminación sobre la mujer, art. 5b). Sin embargo seguía siendo una concepción demasiado amplia, y como observó el delegado de Finlandia, el ISN debería ser la consideración primordial sólo en las medidas en que estuviera implicado su bienestar (E/CN.4/1989/48, parr.123). En efecto, esta es la razón que explica la diversidad con la que el ISN aparece redactado a lo largo de la Convención, tal y como señalábamos unos párrafos más arriba, al hablar de su institucionalización.

En definitiva, como observa el profesor Ph. Alston (1994b), el objetivo implícito al optar por la palabra “a” en detrimento de "la” era asegurar la suficiente flexibilidad, por lo menos en los casos extremos, para permitir que puedan prevalecer otros intereses al margen de los del niño. Sin embargo, por la formulación adoptada parecería imponer-

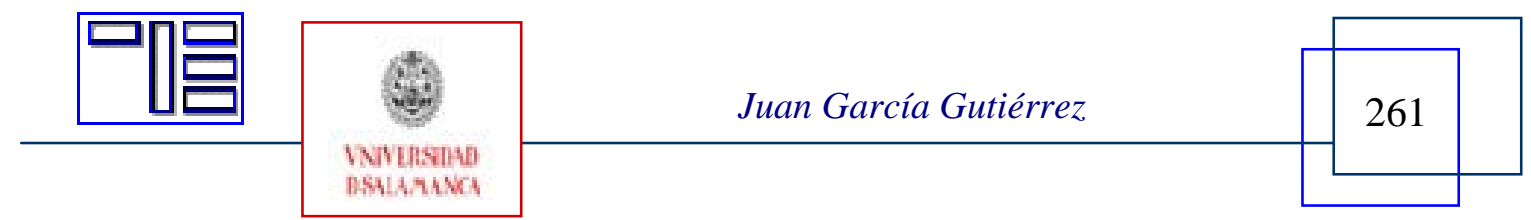


se la carga de la prueba a aquellos que pretenden alcanzar ese resultado "no centrado en el interés del niño”. Esta aparente flexibilidad del principio desaparecería cuando se trata de ámbitos que lo afectan directamente como ocurre en el caso de la adopción, donde se establece que el ISN será “the paramount consideration (Art. 21, CDN)”.

Otro aspecto importante al que hay que prestar atención es la ubicación que se le ha dado al principio en el interior de la Convención, en el artículo tercero, ya sólo algunos poseen además un carácter de “principio rector” de la Convención. En efecto, aunque parezca mentira, escribe el profesor Alston (1994: 11), ninguna delegación se centro especialmente en ello. Esto es, en las consecuencias que se seguirían de la inclusión del principio de ISN en el artículo 3.1, en lugar de haberlo colocado en otro artículo de la Convención, y ello porque en ningún momento durante los trabajos preparatorios se consideraron los efectos de un conflicto de intereses entre un determinado derecho reconocido en la Convención y la interpretación de lo que constituye el interés superior del niño en concreto.

En última instancia, lo que estos aspectos problemáticos (tanto el tema de la indeterminación, como del relativismo cultural y la formalidad de la redacción) ponen de manifiesto son dos formas distintas de entender ISN, o sea, el bien del niño. Mientras que unos tienen una concepción más social del principio, esto es, mediada por la cultura, lo que en cada momento histórico se piensa que es beneficioso o perjudicial para el niño. Para otros, el bien del niño pasa por el reconocimiento de su dignidad intrínseca como ser humano, y por tanto, por una reflexión de lo que ésta afirmación implica para la infancia.

\section{5.- EL CONCEPTO DE EVOLUCIÓN DE LAS FACULTADES DEL NIÑO}

Aunque el reconocimiento de los derechos del niño es uno de los hitos más significativos en el campo de los derechos humanos hay que estar muy atentos a la interpretación que se hace de ellos. Así es necesario tener presente el sentido con que el principio de interés superior del niño se introdujo en la Convención y qué márgenes de interpretación puede tener en cada caso. No pueden interpretarse adecuadamente los derechos del niño sin reflexionar sobre qué significa en cada momento su “interés supe-

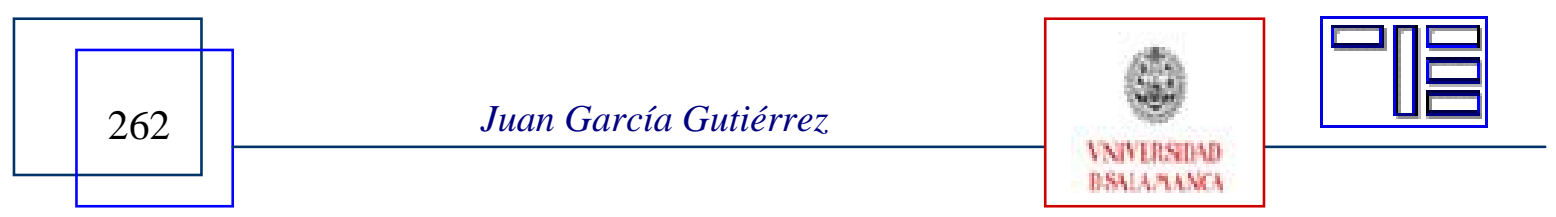


rior”. Así resulta necesario vincular el principio de ISN a la noción de "evolución de las facultades” del niño para lograr una mejor comprensión del bien del niño ${ }^{10}$.

La definición del concepto de evolución de las facultades del niño aparece expresada en el artículo 5 de la CDN:

“Los Estados Partes respetarán las responsabilidades, los derechos y deberes de los padres o, en su caso, de los miembros de la familia ampliada o de la comunidad, según establezca la costumbre local, de los tutores u otras personas encargadas legalmente del niño de impartirle, en consonancia con la evolución de sus facultades, dirección y orientación apropiadas para que el niño ejerza los derechos reconocidos en la presente Convención”.

El concepto de "facultades en evolución" que se encuentra en la base del principio de autonomía y de participación. En concreto, hay que hacer una lectura conjunta de los artículos 12.1, 14.2 de la CDN para completar la formulación que establece el art. 5 de la CDN:

“Los Estados Partes garantizarán al niño que esté en condiciones de formarse un juicio propio el derecho a expresar su opinión libremente en todos los asuntos que afectan al niño, teniéndose debidamente en cuenta las opiniones del niño, en función de la edad y madurez del niño (art. 12.1)”;

“Los Estados Partes respetarán los derechos y deberes de los padres y, en su caso, de los representantes legales, de guiar al niño en el ejercicio de su derecho de modo conforme a la evolución de sus facultades (art. 14.2)”.

Podríamos concluir afirmando como los derechos y libertades de la CDN relacionados con el principio de autonomía dependen de la evolución de las facultades del niño, de su edad, y su madurez para formarse un juicio propio. Así aparecerían vinculados educación, autonomía y juicio propio. En concreto, la formación de un juicio propio se nos revela como una finalidad educativa implícita en la CDN. Así en la CDN el reconocimiento de la autonomía personal para el ejercicio de determinados derechos y libertades descansa en la suposición de que tales individuos poseen la competencia necesaria para efectuar elecciones y tomar decisiones de manera informada y sensata.

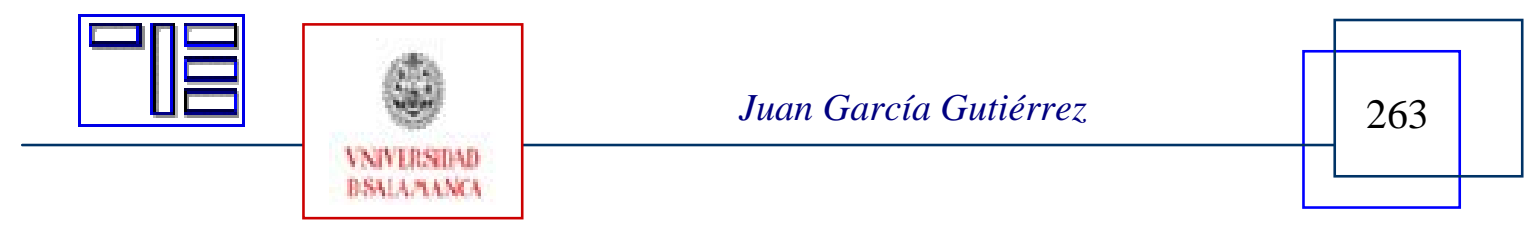


En seguida aparecen una serie de casos, muchos de ellos relacionados con los que podríamos denominar como una “construcción posmoderna de la identidad” (como por ejemplo, el acceso a operaciones de tipo estético, el consentimiento para mantener relaciones sexuales, o el acceso con o sin conocimiento de sus padres a la "píldora del día después”, etc.), la iniciación en una determinada práctica religiosa (en algunos casos “sectas”) o filosofía de vida, etc. Se trata de ámbitos en los que la autonomía del niño en la toma de decisiones puede chocar con su propio interés o el interés de otro niño, o incluso puede presentase un conflicto con su familia y/o progenitores.

\section{1.- Problemas y limitaciones del enfoque de la evolución de las facultades del niño}

Por otra parte, podemos señalar dos problemas o limitaciones que subyacen al enfoque de la evolución facultades del niño en la CDN. En primer lugar la cuestión del relativismo cultural, tal y como aparecía en la noción de interés superior del niño. Por otra parte, la cuestión de los límites de edad.

\section{a) Desarrollo evolutivo v. teorías culturales}

En la investigación sobre la evolución de las facultades del niño que realiza G. Lansdown (2005:26 y ss.) para el Instituto de Investigaciones Innocenti de Unicef, diferencia dos enfoques teóricos contrarios que sirven de base a las reflexiones actuales sobre el desarrollo infantil y, que por tanto, influyen en la comprensión e interpretación del concepto de facultades en evolución de los niños. Tales enfoques son, por un lado, lo que denomina como "teorías convencionales sobre las etapas evolutivas”, y por otro las “teorías culturales”.

Las teorías evolutivas parten del presupuesto de que el desarrollo es un proceso que avanza por etapas, ya sea respecto a las capacidades físicas, morales, sociales, emocionales o intelectuales. Lansdown crítica estas teorías por partir de una posición etnocéntrica. Así, las investigaciones en las cuales se basan las teorías convencionales se originaron en contextos europeos y norteamericanos, y reflejan suposiciones relativas a la infancia tal como se da en dichas sociedades. Según él, estas teorías tienes cinco puntos clave: el desarrollo infantil es un proceso universal; la adultez tiene estatus normati-

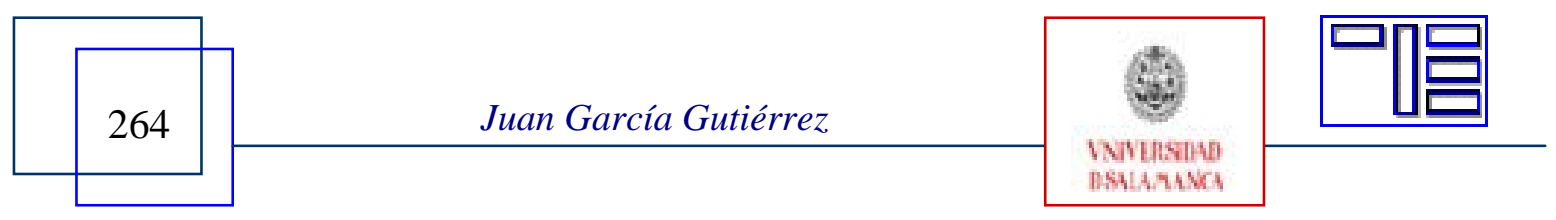


vo; los objetivos del desarrollo son universales; la desviación de la norma representa un peligro para el niño; la niñez es un largo periodo de dependencia, en el cual lo niños son receptores pasivos de la protección, la formación, sabiduría y orientación de los adultos, en vez de contribuir activamente a su ambiente social. Precisamente estos aspectos serán los criticados por las llamadas "teorías culturalistas”.

Progresivamente, señala el propio Lansdow, los expertos en psicología evolutiva han ido elaborando un marco teórico que ve el desarrollo infantil como un proceso cultural y la infancia como el producto de determinados procesos económicos, sociales y culturales. Las teorías culturales reconocen que todos los entornos son resultado de una construcción social y que no hay nada de natural en cualquier modelo individual de crianza de los niños. Así se identifican dentro de los ambientes donde habitan los niños tres elementos que influyen en su desarrollo: los entornos físico y sociales en que viven (la familia, las estructuras sociales y la organización de su vida cotidiana), las costumbres reglamentadas por la cultura y las prácticas de crianza (medidas para el cuidado y la educación, actitudes respecto al juego, la disciplina y la formación), las convicciones o etnoteorías de los padres (objetivos y prioridades del desarrollo del niño y opiniones sobre cómo se pueden cumplir con ellos).

Por lo que se desprende de su trabajo, Lansdown parece decantarse por el segundo enfoque. Algo que a mi juicio no hace sino profundizar y agudizar el relativismo con el que la doctrina se enfrentan a las cuestiones nucleares de los derechos de la infancia. Por otra parte, cabe encontrar en las corrientes culturalistas actuales ciertas influencias o puntos de encuentro entre las ya clásicas teorías marxistas que actualmente ya en desuso necesitan adoptar nuevas formas y nuevas categorías para ejercer la crítica. El problema de ciertos enfoques neo-marxistas (algo que en educación, resulta aún más grave), como estos de corte culturalista es su reduccionismo antropológico al no considerar suficientemente la libertad de la persona.

\section{b) La cuestión de los límites de edad}

Uno de los aspectos que más negativamente influyen en la consideración de las “facultades en evolución” es la amplitud temporal que comprende la definición de "ni-

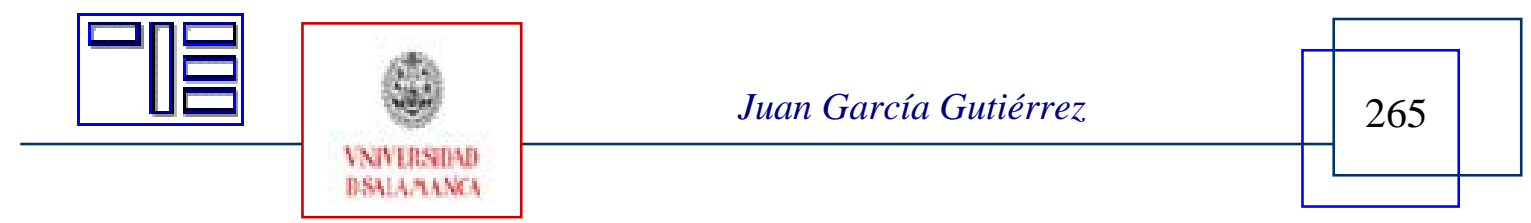


ño" en la $\mathrm{CDN}^{11}$. Así se considera como niño a "todo ser humano menor de dieciocho años de edad, salvo que haya alcanzado antes la mayoría de edad (art. 1)”. El no haber diferenciado tramos de edad o niveles de competencias para el ejercicio de determinados derechos o libertades es uno de los aspectos más negativos de la propia Convención. Así algo tan característico de las legislaciones internas como es fijar unos límites de edad para determinados derechos o libertades es algo que no aparece reflejado en la propia Convención, y los que aparecen se deben a los dos Protocolos adicionales: el "Protocolo facultativo de la Convención sobre los Derechos del Niño relativo a la participación de niños en los conflictos armados (A/RES/45/263, de 25.5.2000)” y el "Protocolo facultativo de la Convención sobre los Derechos del Niño relativo a la venta de niños, la prostitución infantil y la utilización de niños en la pornografía (A/RES/54/264, de 25.6.2000)”. Los derechos y libertades que suelen aparecer vinculados a determinados límites de edad suelen ser los de participación política (voto y representatividad); consentimiento de relaciones sexuales y matrimonio; edad para acceso al trabajo; edad de escolarización; o la de responsabilidad penal ${ }^{12}$.

Por otra parte, y ante la ausencia en la CDN de límites o diferenciación por edades es interesante rescatar alguna iniciativa que ha aparecido recientemente tratando de dar respuesta a esta necesidad de acotar aquello que en la CDN se denomina genéricamente como "niño”. Así cabe destacar la iniciativa española de "Convención Iberoamericana de Derechos de la Juventud ${ }^{13}$ ”. Así, en esta Convención se denomina “joven” o “juventud” a todas las personas, nacionales o residentes en algún país de Iberoamérica, comprendidas entre los 15 y los 24 años de edad (art, 1).

\section{6.- NECESIDAD DE UN ENFOQUE RELACIONAL PARA LOS DERECHOS DE LA INFANCIA: EL APRENDIZAJE DE LA “AUTONOMÍA RELACIO- NAL”}

Desde hace décadas estamos asistiendo a una proliferación de convenciones y declaraciones de derechos humanos que no hacen sino alargar la protección y cobertura que ofrecen los derechos humanos a grupos de población y sectores cada vez más espe-

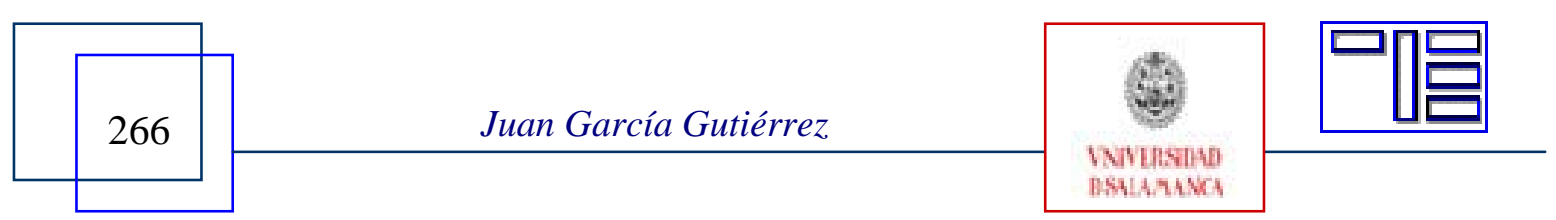


cíficos, algo que en ocasiones multiplica los conflictos y dificulta una correcta interpretación de los mismos.

En este contexto, nos encontramos con que el desarrollo de los derechos de la infancia aparece vinculado históricamente con el proceso de emancipación de la mujer y los movimientos feministas de los siglos XIX y XX. Así tenemos que la concepción del principio de autonomía que late en la Convención y que expresa parte del articulado es una autonomía más política que educativa, como correspondería al tratarse de un ámbito como la infancia. Esto es, se trata de una autonomía que busca más la emancipación individual que el desarrollo propio del niño ${ }^{14}$.

De hecho la calificación como “especialmente grupo vulnerable”, sobre todo cuando se trata de las niñas, debiera llamarnos a reflexionar sobre el papel que la autonomía tiene en el desarrollo del niño o la niña, y como ésta no puede ser interpretada como emancipación ni traspasada del ámbito político al de la infancia sin una reflexión pedagógica previa. Así, tanto la autonomía como el cuidado estarán al servicio del desarrollo y "bienestar social, espiritual y moral, y su salud física y mental (art. 17, CDN)" del niño. Hay que señalar como en muchas ocasiones el niño aparece atrapado en su propia autonomía (B. C. Hafen, 1976; 1996), y hasta educativamente se nos antoja como el objetivo más importante que podemos lograr. Sin embargo, una lectura educativa de la autonomía bien puede pasar por una propuesta pedagógica de la dependencia (F. Bárcena y J. C. Melich, 1999), como proponemos (aunque por espacio no desarrollaremos en este momento) por una "autonomía relacional”.

En efecto, la educación como fenómeno relacional en el que prima el “interés”, pero sobre todo el "bien” del niño es un espacio privilegiado para abordar una lectura relacional de los derechos humanos, y en concreto de los contenidos en la Convención, con el objetivo de superar la clásica tensión entre los principios de autonomía y cuidado que emergen en el articulado. Abogar por un enfoque relacional en la interpretación de los derechos de la infancia significa sobre todo entenderlos en su contexto natural, esto es, como es la familia el espacio natural más apropiado para cultivar el respeto por los derechos humanos y su desarrollo.

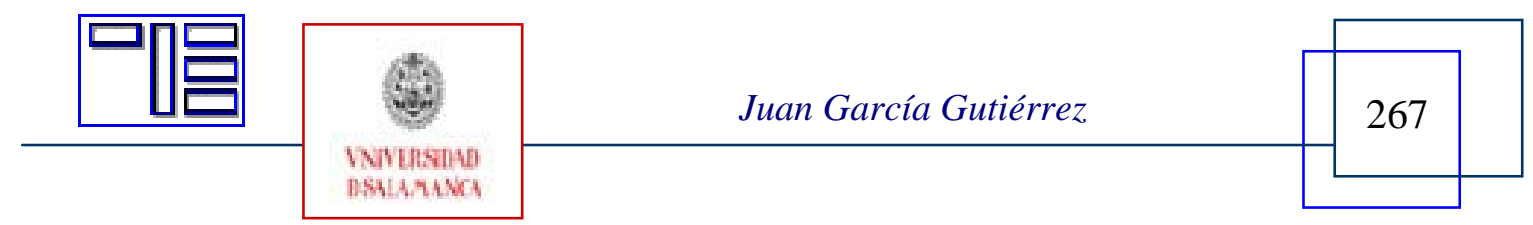




\section{7-REFERENCIAS}

ALSTON, PH. \& STEINER, H. J. (2000). International human rights in context: law, Politics and morals. Oxford: Oxford University Press.

VOSS, I. N. (1993). In the Best Interest: the Adoption of F. H., an Indian child. BYU Journal of Public Law. Vol. 8, 151-171.

ALSTON, PH. (Ed.) (1994). The best interests of the child. Reconciling culture and human rights. Oxford: Clarendon Press.

ALSTON, PH. (1994b) The Best Interests Principle: Towards a Reconciliation of Culture and Human Rights. International Journal of Law and the Family. Vol. 8, 12.

BECCHI, E. (1998). Il mondo dell'infanzia. Storia, cultura, problema. Roma: Laerza.

CHEN, L. (1989). Toward Adoption of the UN CDN: A Policy-Oriented Overview. American Sociological and International Law. Vol. 83, 157-161;

CERDA, J. S. (1990). The Draft Convention on the Rights of the Child: new Rights. Human Rights Quarterly. Vol 12, 115-119;

DETRICK, S. (Ed.) (1992). The UN Convention on the Rights of the Child: a Guide to the “Travaux Préparatoires”. London: Martinus Nijhoff Publihers;

HAFEN, B. C. (1976). Children's Liberation and the New Egalitarianism: some Reservations about Abandoning Youth to their "Rights”. Brigham Young University Law Review. Vol. XX, 605-658;

HAFEN, B. C. (1996). Abandoning Children to their Autonomy: the United Nations Convention on the Rights of the Child. Harvard International Law Journal. Vol. 37, $\mathrm{n}^{\circ}$ 2, 460 .

GERISON LANSDOWN, G. (2005). La evolución de las facultades del niño. Florencia: Centro de Investigaciones Innocenti.

HAFEN, B. C. (1976). Children's Liberation and the New Egalitarianism: some Reservations about Abandoning Youth to their "Rights". Brigham Young University Law Review. Vol. XX, 605-658;

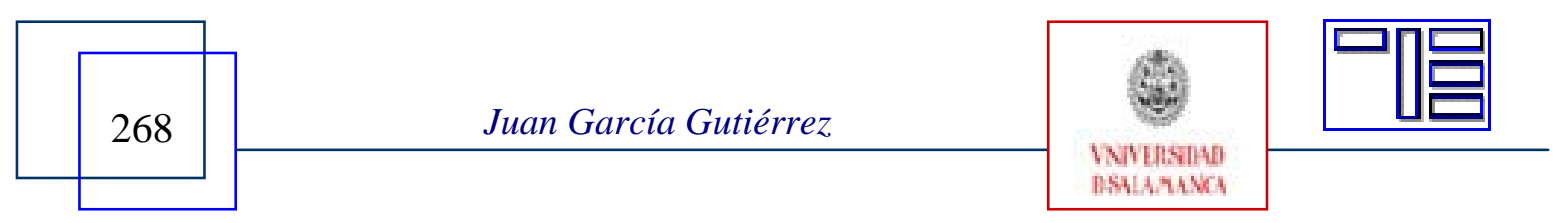


HAFEN, B. C. \& HAFEN, J. O. (1996). Abandoning Children to their Autonomy: the United Nations Convention on the Rights of the Child. Harvard International Law Journal. Vol. 37, n 2, 460.

MCGOLDRICK, D. (1991). The UN Convencion on the Rights of the Child. International Journal of Law and the Family. Vol. 5, 132-169.

SOËTARD, M. (1994). Jean-Jacques Rousseau. En Perspectivas : revista trimestral de educación comparada. Vol. XXIV, nº 3-4, 435-448.

\footnotetext{
${ }^{1}$ Este trabajo reformula y amplia algunas de las ideas contenidas en dos artículos anteriores: "L'Interesse superiore del fanciulo in prospectiva pedagogica. Riflessioni e proposte”. En Prospectiva Educazione Permanente. XXIX: 1, Gennaio-Aprile, 2006, pp. 77-105; y "Una voz para la infancia. Génesis y desarrollo de la noción de "interés superior del niño". En T. Vicente y M. Hernández (Coord.). Los derechos de los niños, responsabilidad de todos. Murcia: Servicio de publicaciones de la Universidad de Murcia, 2007, pp. 173-183

${ }^{2}$ Janusz Korczak presenció la toma de Varsovia por los nazis, y fue obligado a trasladar su orfanato al Geto de Varsovia. El 5 de agosto los soldados alemanes llegaron al Geto para recoger a los 192 huérfanos del orfanato y llevarlos al campo de exterminio de Treblinka, a Korczak le fue ofrecido un indulto que no acepto y de éste modo marcho con los niños sin abandonarlos. En el cementerio Powazki de Varsovia hay un mausoleo conmemorativo.

${ }^{3}$ Previos a la CDN existían ya algunos instrumentos adoptados en el Consejo de Europa, como la European Convention on the Legal Status of Children born out of Wedlock (1978) y la European Convention on the Adoption of Children (1968). Después de la CDN se ha establecido en el Consejo de Europa la European Convention on the Exercise of the Children's Rights (2000) donde se afirma el ISN como finalidad expresa del entero Tratado, "The object of the present Convention is, in the best interests of children, to promote their rights, to grant them procedural rights and to facilitate the exercise of these rights by ensuring that children are, themselves or through other persons or bodies, informed and allowed to participate in proceedings affecting them before a judicial authority”. El último texto importante aparecido en el Consejo de Europa que hace referencia a la infancia es la Convention on Contact concerning Children (2005). Para los desarrollos en la jurisprudencia del Tribunal Europeo de Derechos Humanos vid. Kilkelly, U. (1999) The Child and the European Convention on Human Rights. Brookfield: Ashgate Publishing Company; y también, (2001) The Best of Both Worlds for Children's Rights? Interpreting the European Convention on Human Rights in the Light of the UN Convention on the Rights of the Child. Human Rights Quarterly. Vol. 23, 308-326.

${ }^{4}$ Pese a lo ya escandaloso de la pena de muerte, la Declaración Árabe de Derechos Humanos (1994) prevé que la pena de muerte no puede ser ejecutada a menores de dieciocho años, ni a una mujer embarazada hasta dar a luz, o a una mujer con hijos menores de dos años desde el parto (art. 12). Liga Árabe [14.6.2005] http://www.arableagueonline.org.

${ }^{5}$ Organización para la Unidad Africana [19.6.2006]: http://www.africa-union.org. La Organización de la Unidad Africana también tiene sus propios instrumentos específicos para la infancia, la Declaración de Derechos y el Bienestar del niño (1979); y la Carta Africana de Derechos y Bienestar del Niño (1999).

6 "Los Estados Partes adoptarán todas las medidas administrativas, legislativas y de otra índole para dar efectividad a los derechos reconocidos en la presente Convención. En lo que respecta a los derechos económicos, sociales y culturales, los Estados Partes adoptarán esas medidas hasta el máximo de los recursos de que dispongan y, cuando sea necesario, dentro del marco de la cooperación internacional (CDN, art. 4)".

${ }^{7}$ Otras conceptualizaciones del ISN aparecen en los artículos: 9.1; art. 9.3; art. 18.2; art. 20.1; y art. 37 de la CDC.
}

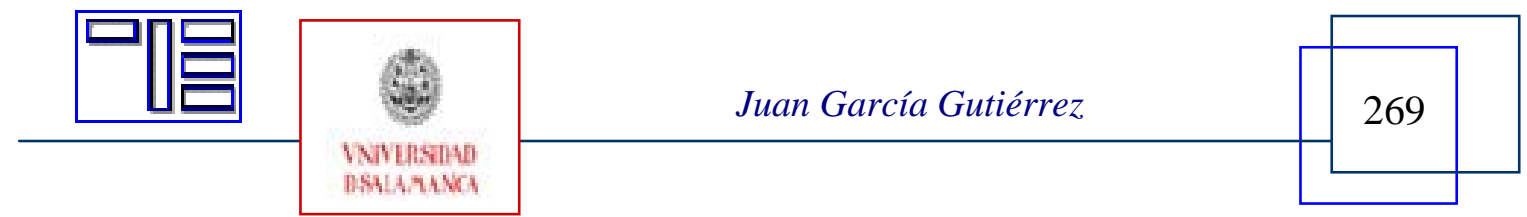


Revista Electrónica Teoría de la Educación.

Educación y Cultura en la Sociedad de la Información.

http://www.usal.es/teoriaeducacion

Vol. 9. No1.Febrero 2008

${ }^{8}$ La cuestión de la indeterminación jurídica ha sido principalmente estudiada por R. H. Mnookin (1975) Child-Custody Adjudication: Judicial Functions in the Face of Indeterminacy. Law and Contemporary Problems. Vol. 39, $\mathrm{n}^{\circ}$ 3, 227-294; y J. Eekelaar (1994) Interests of the Child and the Child's Wishes: the role of dynamic self-determinism. International journal of Law and the Family. Vol. 8, 42-61; J. Eekelaar (1992) The Importance of Thinking that Children have Rights. International journal of Law and the Family. Vol. 6, 221-235.

${ }^{9}$ Estos factores también afectan a la noción de "evolución de las facultades del niño" donde se debate se centra entre un enfoque evolutivo o culturalista. Vid.G. Lansdown (2005). The evolving capacities of the child. Florencia: Innocenti Insigh.

${ }^{10}$ Vid. Gerison Lansdown, G. (2005). La evolución de las facultades del niño. Florencia: Centro de Investigaciones Innocenti.

${ }^{11}$ Un aspecto negativo y controvertido de la Convención es la ausencia de protección que tiene el niño aun no nacido. Es necesario recordar como el Preámbulo de la CDN señala, recordando la Declaración del '59, que "el niño, por su falta de madurez física y mental, necesita protección y cuidados especiales, incluso la debida protección legal, tanto antes como después de su nacimiento". En este sentido, y desde la perspectiva de la evolución de las facultades, llama la atención la escasa consideración que la CDN presta al momento anterior al nacimiento y que constituye, sin lugar a dudas, un momento crítico en la evolución de las facultades del niño. Precisamente, cabría hacer caso de los avances en la pediatría, y en la estimulación temprana intrauterina para que el grado de protección jurídica sea proporcional al de dependencia y vulnerabilidad. Y esto no sólo en el momento de la infancia (tanto antes como después del parto) sino extensible a todas aquellas situaciones de dependencia en las que la persona dependiente carece de una protección y cuidado proporcional a su dependencia. Vid. Alston, Ph. (1990).The Unborn Child and Abortion under the Draft Convention on the Rights of the Child. En Human Rights Quarterly, 12: 1, pp. 156-178; Nuñez, T. (2003). ¿Qué es un niño?. Una visión desde el derecho internacional público. En Revista Española de Educación Comparada, 9, 13-47.

${ }^{12}$ Una Tabla sobre edades mínimas de salida de la escuela, acceso al trabajo, consentimiento en el matrimonio y edad penal por países aparece en la página web de K. Tomasevski (ex-Relatora de Naciones Unidas para el Derecho a la Educación), [7.10.2007] http://www.right-toeducation.org/content/age/table_esp.html.

${ }^{13}$ Vid. [7.10.2007] http://convencion.oij.org/, para más información puede verse la web de la Organización Iberoamericana de la Juventud: http://www.oij.org.

${ }^{14}$ Se trata de una visión política de la autonomía frente a una visión educativa. Tal y como lo plantea Arendt, "la educación no debe tener un papel en la política, porque en la política siempre tratamos con personas que ya están educadas (p. 188)”. Arendt, H. (1996) Entre el pasado y el futuro. Barcelona: Península.

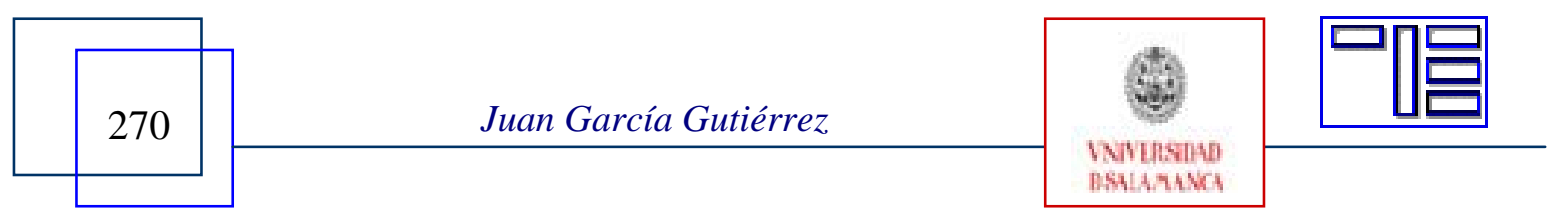

